International Journal of Modern Physics D

(C) World Scientific Publishing Company

\title{
Beyond Gravitoelectromagnetism: Critical Speed in Gravitational Motion
}

\author{
Bahram Mashhoon \\ Department of Physics and Astronomy, University of Missouri-Columbia, \\ Columbia, Missouri 65211, USA \\ mashhoonb@missouri.edu \\ Received Day Month Year \\ Revised Day Month Year \\ Communicated by Managing Editor
}

\begin{abstract}
A null ray approaching a distant astronomical source appears to slow down, while a massive particle speeds up in accordance with Newtonian gravitation. The integration of these apparently incompatible aspects of motion in general relativity is due to the existence of a critical speed. Dynamics of particles moving faster than the critical speed could then be contrary to Newtonian expectations. Working within the framework of gravitoelectromagnetism, the implications of the existence of a critical speed are explored.

The results are expected to be significant for high energy astrophysics.
\end{abstract}

Keywords: General relativity; critical speed; tidal acceleration.

\section{Introduction}

Gravitoelectromagnetism ("GEM") originates from the similarity between Coulomb's law of electricity and Newton's law of universal gravitation. In the course of the development of his dynamical theory of the electromagnetic field, Maxwell considered a similar theory for the gravitational field ${ }^{1}$. He noted that gravitational charges are all of the same kind and lead to attraction instead of repulsion. On the basis of certain energy arguments, he then concluded that one could not arrive at a fundamental theory of gravitation in this way ${ }^{1}$. Several years later, difficulties with the excess perihelion motion of Mercury led to the phenomenological introduction of a gravitomagentic force due to mass current in the Sun ${ }^{2,3}$. However, in 1915 Einstein's general relativity provided a beautiful explanation of Mercury's motion ${ }^{4}$. This involved a relativistic correction to the Newtonian gravitoelectric potential of the Sun. Soon afterwards, the gravitational influence of the rotation of the Sun on planetary orbits was determined within general relativity by de Sitter ${ }^{5}$. This equatorial gravitomagnetic precession turned out to be much smaller and in the opposite sense as the Einstein gravitoelectric precession ${ }^{5}$.

A field theory that successfully combines Newtonian gravitation with Lorentz invariance should necessarily contain a gravitomagnetic field ${ }^{6}$. The first general investigation of the gravitomagnetic field within the framework of general relativity 
is due to Thirring ${ }^{7}$. Moreover, the general rate of precession of the orbit of a test particle in the field of a rotating mass was determined by Lense and Thirring ${ }^{8}$. An English translation of the Thirring-Lense papers together with a critical commentary on their results has been provided in Ref. 9. Extensive reviews and discussions of the various aspects of GEM are contained in Refs. $10-12$. On the observational side, there has been progress, especially since NASA's GP-B was launched on April $20,2004 .^{13-15}$

The GEM framework will be employed in this paper in order to illustrate the existence of a critical speed in the dynamics of a test particle moving in a given GEM field. There are two principal approaches to GEM. The linear perturbation approach to GEM is briefly presented in Section 2 and the general equation of motion of a test particle is investigated in Section 3. It is shown that this equation of motion contains a critical speed given by $v_{c}=c / \sqrt{3}$ in the case of one-dimensional motion. Section 4 is devoted to a more basic invariant treatment of the critical speed; for this purpose, the spacetime curvature approach to GEM is employed using the quasiinertial Fermi coordinates. In this invariant analysis of relative motion, the critical speed turns out to be $V_{c}=c / \sqrt{2}$. For relative ("ultrarelativistic") motion that is faster than this critical speed, the results of recent investigations of tidal acceleration/deceleration phenomena in the field of a neutron star or a black hole are briefly summarized. The case of ultrarelativistic fluid flow is very similar and the relevant equations are derived in Appendix A. Finally, Section 5 contains general remarks regarding the gravitational acceleration/deceleration of ultrarelativistic particles.

\section{Motion in a GEM Field}

Gravitoelectromagnetism provides a useful method for the description of the gravitational field generated by a slowly rotating "nonrelativistic" astronomical source in the linear approximation of general relativity ${ }^{4}$. The spacetime metric can be written as $g_{\mu \nu}=\eta_{\mu \nu}+h_{\mu \nu}$, where $\eta_{\mu \nu}$ is the Minkowski metric tensor with signature +2 and $h_{\mu \nu}$ is a first-order perturbation. The gravitational potentials are in general gauge-dependent, i.e. $h_{\mu \nu} \rightarrow h_{\mu \nu}+\epsilon_{\mu, \nu}+\epsilon_{\nu, \mu}$, where $\epsilon_{\mu}$ is a vector associated with the choice of the background inertial coordinates $x^{\mu}=(c t, \mathbf{x})$. In terms of the trace-reversed potentials $\bar{h}_{\mu \nu}=h_{\mu \nu}-\frac{1}{2} \eta_{\mu \nu} h$, where $h=\operatorname{tr}\left(h_{\mu \nu}\right)$, the gravitational field equations can be written as

$$
\square \bar{h}_{\mu \nu}=-\frac{16 \pi G}{c^{4}} T_{\mu \nu}
$$

after the ("Lorentz") gauge condition $\bar{h}^{\mu \nu}{ }_{, \nu}=0$ has been imposed.

The general retarded solution of (1) involves the particular solution

$$
\bar{h}_{\mu \nu}=\frac{4 G}{c^{4}} \int \frac{T_{\mu \nu}\left(c t-\left|\mathbf{x}-\mathbf{x}^{\prime}\right|, \mathbf{x}^{\prime}\right)}{\left|\mathbf{x}-\mathbf{x}^{\prime}\right|} d^{3} x^{\prime}
$$

plus a solution of the homogeneous wave equation that we simply ignore in the present analysis. For the sources under consideration, $\bar{h}_{00}=4 \Phi / c^{2}, \bar{h}_{0 i}=-2 A_{i} / c^{2}$ 
and $\bar{h}_{i j}=O\left(c^{-4}\right)$. Here $\Phi(t, \mathbf{x})$ is the gravitoelectric potential and $\mathbf{A}(t, \mathbf{x})$ is the gravitomagnetic vector potential. All terms of $O\left(c^{-4}\right)$ will be neglected in the metric tensor. Thus the important quantities of interest in equation (2) are $T^{00}=\rho c^{2}$ and $T^{0 i}=c j^{i}$, where $j^{\mu}=(c \rho, \mathbf{j})$ is the mass-energy current. The conservation of the mass-energy of the source is assured through the imposition of the gauge condition, i.e. $j^{\mu}{ }_{, \mu}=0$ follows from

$$
\frac{1}{c} \frac{\partial \Phi}{\partial t}+\nabla \cdot\left(\frac{1}{2} \mathbf{A}\right)=0 .
$$

The spacetime metric is thus given by

$$
-d s^{2}=-c^{2}\left(1-2 \frac{\Phi}{c^{2}}\right) d t^{2}-\frac{4}{c}(\mathbf{A} \cdot d \mathbf{x}) d t+\left(1+2 \frac{\Phi}{c^{2}}\right) \delta_{i j} d x^{i} d x^{j} .
$$

It is possible to define the gravitoelectric and gravitomagnetic fields in close analogy with electrodynamics

$$
\mathbf{E}=-\nabla \Phi-\frac{1}{c} \frac{\partial}{\partial t}\left(\frac{1}{2} \mathbf{A}\right), \quad \mathbf{B}=\nabla \times \mathbf{A} .
$$

It follows from equations (3) and (5) that

$$
\nabla \times \mathbf{E}=-\frac{1}{c} \frac{\partial}{\partial t}\left(\frac{1}{2} \mathbf{B}\right), \quad \nabla \cdot\left(\frac{1}{2} \mathbf{B}\right)=0,
$$

while the gravitational field equations (1) reduce to

$$
\nabla \cdot \mathbf{E}=4 \pi G \rho, \quad \nabla \times\left(\frac{1}{2} \mathbf{B}\right)=\frac{1}{c} \frac{\partial}{\partial t} \mathbf{E}+\frac{4 \pi G}{c} \mathbf{j} .
$$

These are the Maxwell equations for the GEM field; they are based on a certain convention that is explained in the following paragraph.

To preserve the electromagnetic analogy as much as possible, it would be convenient to be able to employ in the GEM case the standard results of electrodynamics using a special convention. To this end, we assume that the source has gravitoelectric charge $Q_{E}=G M$ and gravitomagnetic charge $Q_{B}=2 G M$, where $M$ is the total mass of the source. It follows that the gravitomagnetic dipole moment of the source is $G \mathbf{J} / c$, where $\mathbf{J}$ is the total angular momentum of the source. Thus far from the source $\left(r=|\mathbf{x}| \gg G M / c^{2}\right)$

$$
\Phi \sim \frac{G M}{r}, \quad \mathbf{A} \sim \frac{G}{c} \frac{\mathbf{J} \times \mathbf{x}}{r^{3}},
$$

which are consistent with equations (2) and (3). Moreover, a test particle of inertial mass $m$ has gravitoelectric charge $q_{E}=-m$ and gravitomagnetic charge $q_{B}=-2 m$ in this convention. We note that the signs of $\left(q_{E}, q_{B}\right)$ are opposite to those of $\left(Q_{E}, Q_{B}\right)$ to preserve the attractive nature of gravity; furthermore, the ratio of gravitomagnetic charge to the gravitoelectric charge is always 2 , since the linear approximation of general relativity involves a spin-2 field. This is consistent with the fact that the ratio of the magnetic charge to the electric charge of a particle is 
unity in Maxwell's spin-1 electrodynamics ${ }^{16}$. The magnetic charge employed here should be distinguished from the magnetic monopole strength, which is strictly zero throughout this paper.

The geodesic equation of motion of a test particle in the stationary GEM field of a source with $\partial \Phi / \partial t=0$ and $\partial \mathbf{A} / \partial t=0$ may be written in a form analogous to the Lorentz force law,

$$
m \frac{d \mathbf{v}}{d t}=-m \mathbf{E}-2 m \frac{\mathbf{v}}{c} \times \mathbf{B}
$$

when velocity-dependent terms of order higher than $v / c$ are neglected. To go beyond the GEM analogy, let us explore the significance of the terms that have been neglected in equation (9). The geodesic equation for a particle with proper time $\tau$ and four-velocity $u^{\mu}=d x^{\mu} / d \tau=\gamma(1, \mathbf{v} / c)$ is given by

$$
\frac{d u^{\mu}}{d \tau}+\Gamma_{\rho \sigma}^{\mu} u^{\rho} u^{\sigma}=0
$$

Once the linear GEM field is given, it is possible to employ equation (10) for the motion of particles of any speed in this field; for instance, one may consider the force-free motion of cosmic rays in the gravitational field of the Earth. For the sake

of simplicity, we choose units such that $c=1$ in what follows. For the GEM field, the Christoffel symbols are

$$
\begin{aligned}
& \Gamma_{0 \mu}^{0}=-\Phi_{, \mu}, \quad \Gamma_{i j}^{0}=2 A_{(i, j)}+\delta_{i j} \Phi_{, 0}, \\
& \Gamma_{00}^{i}=-\Phi_{, i}-2 A_{i, 0}, \quad \Gamma_{0 j}^{i}=\delta_{i j} \Phi_{, 0}+\epsilon_{i j k} B^{k}, \\
& \Gamma_{j k}^{i}=\delta_{i j} \Phi_{, k}+\delta_{i k} \Phi_{, j}-\delta_{j k} \Phi_{, i} .
\end{aligned}
$$

The physical consequences of the geodesic equation (10) with the connection given by equations (11)-(13) are explored in the following section.

\section{Critical Speed}

It is straightforward to show that the components of equation (10) can be expressed as

$$
\begin{aligned}
\frac{1}{\gamma} \frac{d \gamma}{d t}= & \left(1-v^{2}\right) \Phi_{, 0}+2 v^{i}\left[\Phi_{, i}-A_{(i, j)} v^{j}\right], \\
\frac{d v^{i}}{d t}= & \left(1+v^{2}\right) \Phi_{, i}-2(\mathbf{v} \times \mathbf{B})_{i}+2 A_{i, 0}-v^{i}\left(3-v^{2}\right) \Phi_{, 0} \\
& +2 v^{i} v^{j}\left[A_{(j, k)} v^{k}-2 \Phi_{, j}\right] .
\end{aligned}
$$

Here we have used the fact that $d u^{\mu} / d \tau=\gamma d u^{\mu} / d t$ and then separated the equations for $\gamma$ and $\mathbf{v}$. The worldline of the test particle is timelike; therefore, it follows from $u^{\mu} u_{\mu}=-1$ that

$$
\frac{1}{\gamma^{2}}=1-v^{2}-2\left(1+v^{2}\right) \Phi+4 \mathbf{v} \cdot \mathbf{A} .
$$


Consider a stationary axisymmetric source such that for $r \gg G M / c^{2}$ the potentials are given by equation (8). In this case, equation (15) may be expressed as

$$
\begin{aligned}
\frac{d \mathbf{v}}{d t}= & -\frac{G M}{r^{3}}\left[\left(1+v^{2}\right) \mathbf{x}-4(\mathbf{x} \cdot \mathbf{v}) \mathbf{v}\right]-\frac{2 G}{r^{5}}\left[r^{2} \mathbf{J} \times \mathbf{v}\right. \\
& -3(\mathbf{J} \cdot \mathbf{x}) \mathbf{L}+3(\mathbf{J} \cdot \mathbf{L})(\mathbf{x} \cdot \mathbf{v}) \mathbf{v}],
\end{aligned}
$$

where $\mathbf{L}=\mathbf{x} \times \mathbf{v}$. For one-dimensional motion along the rotation axis of the source, equation (17) reduces to

$$
\frac{d \mathbf{v}}{d t}=-\frac{G M \mathbf{x}}{r^{3}}\left(1-3 v^{2}\right)
$$

This equation contains a critical speed $v_{c}=1 / \sqrt{3}$; that is, for motion with $v<v_{c}$, we have the standard attractive force of gravity familiar from Newtonian physics, while for $v=v_{c}$, the particle experiences no force and for $v>v_{c}$ the gravitational attraction turns to repulsion. These results are valid in the linear approximation for the gravitational field under consideration here. It is interesting to note that if we use instead the standard post-Newtonian approximation scheme for the field of the source, the factor $\left(1-3 v^{2}\right)$ in equation (18) becomes $\left(1-3 v^{2}-4 \Phi\right)$, where $\Phi=G M / r \ll 1 .^{17}$

Equation (18) has the exact solution $(v=d r / d t)$

$$
v^{2}=v_{c}^{2}-\left(v_{c}^{2}-v_{\infty}^{2}\right) e^{-6 \Phi}
$$

however, in the linear approximation, $e^{-6 \Phi} \approx 1-6 \Phi$, and equation (19) is valid only in the form

$$
v^{2}=v_{\infty}^{2}+\frac{2 G M}{r}\left(1-3 v_{\infty}^{2}\right),
$$

so that the speed of an infalling test particle increases, remains constant or decreases depending on whether $v_{\infty}$ is less than, equal to or more than $v_{c}=1 / \sqrt{3}$, respectively. In the nonrelativistic limit, $1-3 v_{\infty}^{2} \rightarrow 1$ and equation (20) reduces to the standard result of Newtonian gravitation.

It is crucial to recognize here that highly relativistic outflows in the form of astrophysical jets are expected to occur along the rotation axis of the central massive source. Thus an equation such as (18), though valid for motion along the rotation axis, could nevertheless play a rather significant role in the gravitational physics of phenomena associated with jets.

In connection with jets, it is useful to consider radial motion in the exterior Kerr spacetime. The Kerr metric is given by

$$
\begin{aligned}
-d s^{2}= & -d t^{2}+\Sigma\left(\frac{1}{\Delta} d \hat{\rho}^{2}+d \vartheta^{2}\right)+\left(\hat{\rho}^{2}+a^{2}\right) \sin ^{2} \vartheta d \phi^{2} \\
& +2 G M \frac{\hat{\rho}}{\Sigma}\left(d t-a \sin ^{2} \vartheta d \phi\right)^{2},
\end{aligned}
$$


where $\Sigma=\hat{\rho}^{2}+a^{2} \cos ^{2} \vartheta$ and $\Delta=\hat{\rho}^{2}-2 G M \hat{\rho}+a^{2}$ in Boyer-Lindquist coordinates $(t, \hat{\rho}, \vartheta, \phi)$. Here $M$ is the mass and $a=J / M$ is the specific angular momentum of the source. The geodesic equations of motion for a test particle moving along the rotation axis reduce to

$$
\frac{d t}{d \tau}=\hat{\gamma} \frac{\hat{\rho}^{2}+a^{2}}{\hat{\rho}^{2}-2 G M \hat{\rho}+a^{2}}, \quad \frac{d \hat{\rho}}{d \tau}= \pm \sqrt{\hat{\gamma}^{2}-1+\frac{2 G M \hat{\rho}}{\hat{\rho}^{2}+a^{2}}}
$$

where $\hat{\gamma}$ is an integration constant. When $\hat{\gamma} \geq 1$, it is the Lorentz factor of the test particle as measured by the static inertial observers at spatial infinity, i.e. $\hat{\gamma}^{-2}=1-v_{\infty}^{2}$. It follows from system (22) that

$$
\left(\frac{d \hat{\rho}}{d t}\right)^{2}=v_{\infty}^{2}\left(1+12 \frac{G^{2} M^{2}}{\hat{\rho}^{2}}\right)+\frac{2 G M}{\hat{\rho}}\left(1-3 v_{\infty}^{2}-\frac{4 G M}{\hat{\rho}}\right)+O\left(\frac{1}{\hat{\rho}^{3}}\right) .
$$

To compare with our GEM results, this equation must be expressed in terms of the isotropic radial coordinate $r$,

$$
\hat{\rho}=r\left(1+\frac{G M}{2 r}\right)^{2}
$$

Thus equation (20) can be recovered, in terms of either $\hat{\rho}$ or $r$, to linear order in the gravitoelectric potential.

Shapiro's radar echo delay experiments have demonstrated that photons in effect slow down in the gravitational field of a mass $M$. In Newtonian gravity, however, massive test particles speed up as they approach a gravitational source. It turns out that this is also the case in general relativity if the speed of the particle at infinity is below a critical speed. Otherwise, as $v_{\infty}$ approaches the speed of light the particle has lightlike behavior. That a particle with an initial speed $v_{\infty}$ above a critical speed slows down in the Schwarzschild field as the particle radially approaches the source was first demonstrated in Refs. 18 and 19. The critical speed $v_{c}=1 / \sqrt{3}$ was discussed by Carmeli $^{18}$, who derived equation (20) for radial motion in the exterior Schwarzschild field. The propulsion aspects of this result have recently received attention. ${ }^{20}$ Using a more invariant approach, Jaffe and Shapiro ${ }^{19}$ discussed a transition velocity, $v_{t} \simeq 1 / \sqrt{2}$, in connection with the radial motion of particles in the Schwarzschild field. They argued that the coordinate speed should be replaced with $d l / d t$, where $d l^{2}=\gamma_{i j} d x^{i} d x^{j}$ and $\gamma_{i j}=g_{i j}-g_{0 i} g_{0 j} / g_{00}$. This results, working to linear order in the Schwarzschild spacetime, in an equation of the same form as equation (20) except that the factor $\left(1-3 v_{\infty}^{2}\right)$ is replaced by $\left(1-2 v_{\infty}^{2}\right)$.

It is clear that by employing physically admissible coordinate systems one can obtain different numerical values for the critical speed. The critical speeds $1 / \sqrt{3}$ and $1 / \sqrt{2}$ have also been discussed in Ref. 21. Nevertheless, it is important to remark here that with respect to the basic class of static (generally noninertial) observers in the exterior Schwarzschild spacetime, the local speed of an infalling particle monotonically increases toward unity, while the local speed of light is always equal to unity. 
In the standard post-Newtonian approximation scheme, one is limited to the slow-motion weak-field regime and therefore the coordinate quantities in the equations of motion are assumed to be physically significant as they are observable in the standard interpretation. Here, however, we have been using the linear postNewtonian scheme for the determination of the field generated by the central source, while the test particles can travel at any speed. Thus to study further the predictions of general relativity in this case, we must construct appropriate invariants that would represent actually measurable quantities. To this end, we find it convenient to study relative motion in the quasi-inertial Fermi coordinate system ${ }^{22}$ established about the motion of a reference observer. This manifestly invariant approach is described in the next section.

\section{Fermi Coordinates}

Consider an observer $\mathcal{O}$ following a geodesic in the gravitational field of an astronomical source. Let $\lambda_{(\alpha)}^{\mu}$ be the observer's local orthonormal tetrad frame that is parallel propagated along the worldline of $\mathcal{O}$. That is, $\lambda_{(0)}^{\mu}=d x^{\mu} / d \tau$ is the observer's four-velocity vector as well as its local temporal axis and $\lambda^{\mu}{ }_{(i)}, i=1,2,3$ are the spatial unit gyro directions that form the local spatial frame of the observer. Here $\tau$ is the proper time of $\mathcal{O}$. To describe physical phenomena relative to $\mathcal{O}$, it is natural to construct a Fermi coordinate system along its worldline. This is a geodesic coordinate system based on the observer's local frame. Indeed, an event $P$ with Fermi coordinates $X^{\mu}=(T, \mathbf{X})$ can be orthogonally connected to the worldline of the reference observer at $P_{0}$ by a unique spacelike geodesic of proper length $\sigma$. Let $\tau$ be the proper time along the reference trajectory at $P_{0}$ and $\eta^{\mu}=\left(d x^{\mu} / d \sigma\right)_{0}$ be the unit tangent vector to the spacelike geodesic at $P_{0}$; then, $T=\tau$ and $X_{i}=\sigma \eta_{\mu} \lambda^{\mu}{ }_{(i)}$. Thus, $\mathcal{O}$ has Fermi coordinates $(\tau, \mathbf{0})$, so that the reference observer always occupies the spatial origin of the Fermi coordinate system. The spacetime metric in Fermi coordinates is given by

$$
\begin{aligned}
& { }^{F} g_{00}=-1-{ }^{F} R_{0 i 0 j}(T) X^{i} X^{j}+\ldots, \\
& { }^{F} g_{0 i}=-\frac{2}{3}{ }^{F} R_{0 j i k}(T) X^{j} X^{k}+\ldots, \\
& { }^{F} g_{i j}=\delta_{i j}-\frac{1}{3}{ }^{F} R_{i k j l}(T) X^{k} X^{l}+\ldots,
\end{aligned}
$$

where

$$
{ }^{F} R_{\alpha \beta \gamma \delta}(T)=R_{\mu \nu \rho \sigma} \lambda^{\mu}{ }_{(\alpha)} \lambda_{(\beta)}^{\nu} \lambda_{(\gamma)}^{\rho} \lambda_{(\delta)}^{\sigma}
$$

is the projection of the Riemann tensor on the local tetrad frame of observer $\mathcal{O}$. The Fermi coordinates are admissible in a cylindrical region with $|\mathbf{X}|<\mathcal{R}$ along the reference worldline such that $\mathcal{R}(T)$ is a certain minimum radius of curvature of spacetime. 
The equation of motion of a free test particle in the Fermi coordinate system can be expressed as

$$
\frac{d U^{\mu}}{d s}+{ }^{F} \Gamma_{\alpha \beta}^{\mu} U^{\alpha} U^{\beta}=0,
$$

where $U^{\mu}=d X^{\mu} / d s=\Gamma(1, \mathbf{V})$ is the particle's four-velocity. The geodesic motion of the particle is always timelike; therefore,

$$
\Gamma^{-2}=-{ }^{F} g_{00}-2{ }^{F} g_{0 i} V^{i}-{ }^{F} g_{i j} V^{i} V^{j}>0 .
$$

It follows from equation (29) that

$$
\begin{aligned}
& \frac{1}{\Gamma} \frac{d \Gamma}{d T}=-{ }^{F} \Gamma_{\alpha \beta}^{0} \frac{d X^{\alpha}}{d T} \frac{d X^{\beta}}{d T}, \\
& \frac{d^{2} X^{i}}{d T^{2}}+\left({ }^{F} \Gamma_{\alpha \beta}^{i}-{ }^{F} \Gamma_{\alpha \beta}^{0} V^{i}\right) \frac{d X^{\alpha}}{d T} \frac{d X^{\beta}}{d T}=0 .
\end{aligned}
$$

The equation of relative motion (32) can be expressed to linear order in distance away from the reference observer $\mathcal{O}$ by using the terms given explicitly in the metric tensor (25)-(27). The result is

$$
\begin{aligned}
& \frac{d^{2} X^{i}}{d T^{2}}+{ }^{F} R_{0 i 0 j} X^{j}+2{ }^{F} R_{i k j 0} V^{k} X^{j} \\
& \quad+\frac{2}{3}\left({ }^{F} R_{0 k j 0} V^{i} V^{k}+{ }^{F} R_{i k j l} V^{k} V^{l}+{ }^{F} R_{0 k j l} V^{i} V^{k} V^{l}\right) X^{j}=0
\end{aligned}
$$

while the modified Lorentz factor is given by

$$
\begin{aligned}
\frac{1}{\Gamma^{2}}= & 1-V^{2}+{ }^{F} R_{0 i 0 j} X^{i} X^{j}+\frac{4}{3}{ }^{F} R_{0 j i k} X^{j} V^{i} X^{k} \\
& +\frac{1}{3}{ }^{F} R_{i k j l} V^{i} X^{k} V^{j} X^{l}
\end{aligned}
$$

Neglecting the velocity-dependent terms in equation (33), one recovers the Jacobi equation. It is important to note that the treatment of test particle motion can be generalized to the flow of a perfect fluid as in Appendix A.

To interpret the generalized Jacobi equation (33), let us first proceed by analogy with the linear perturbation theory of Section 2 and note that the metric in the Fermi frame reduces to the Minkowski metric along the reference trajectory; hence, one may write ${ }^{F} g_{00}=-1+2 \Phi$ and ${ }^{F} g_{0 i}=-2 A_{i}$, where

$$
\Phi=-\frac{1}{2}{ }^{F} R_{0 i 0 j} X^{i} X^{j}, \quad A_{i}=\frac{1}{3}{ }^{F} R_{0 j i k} X^{j} X^{k} .
$$

Moreover, the corresponding GEM fields are obtained from equation (5), so that to lowest order

$$
E_{i}={ }^{F} R_{0 i 0 j} X^{j}, \quad B_{i}=-\frac{1}{2} \epsilon_{i j k}{ }^{F} R_{j k 0 l} X^{l} .
$$

It follows that equation (33) can be written as

$$
m \frac{d \mathbf{V}}{d T}=q_{E} \mathbf{E}+q_{B} \mathbf{V} \times \mathbf{B}+O\left(V^{2}\right),
$$


where $q_{E}=-m$ and $q_{B}=-2 m$ as before. Though this analog of the Lorentz force law is extremely interesting and leads to the physical interpretation of the Bel and Bel-Robinson tensors ${ }^{23}$, it is important to recognize that the higher-order terms in equation (37) become rather significant for ultrarelativistic motion. To see this, let us imagine a circumstance where one-dimensional motion - along the $Z$ direction, say - is possible. Then it follows from the symmetries of the Riemann tensor that equation (33) reduces to

$$
\frac{d^{2} Z}{d T^{2}}+k(T)\left(1-2 \dot{Z}^{2}\right) Z=0
$$

where $\dot{Z}=d Z / d T$ and $k(T)={ }^{F} R_{T Z T Z}$. The generalized Jacobi equation (38) has solutions for $\dot{Z}= \pm 1 / \sqrt{2}$ such that the relative motion within the Fermi frame is uniform. Below the critical speed $V_{c}=1 / \sqrt{2}$, the relative motion is similar to what is expected from relativistic tides in accordance with the Jacobi equation. The situation changes drastically, however, for ultrarelativistic relative motion above the critical speed $V_{c}$. If $k(T)<0$, then an initially ultrarelativistic particle decelerates with respect to the reference particle and asymptotically approaches uniform motion with critical speed $V_{c}$. If $k(T)>0$, then an initially ultrarelativistic particle accelerates with respect to the reference particle. ${ }^{24}$ These results for the motion of free particles can be extended to fluid flow (see Appendix A).

Ultrarelativistic particles are expected to be produced in abundance in the central engines of active galactic nuclei as well as supernova explosions and $X$-ray sources. It is therefore natural to expect that the tidal acceleration/deceleration of ultrarelativistic particles could have significant implications for the physics of astrophysical jets and high-energy cosmic rays.

Assuming that the gravitational field of the central source may be described by the Kerr field, consider a Fermi coordinate system established along reference escape trajectories on the axis of rotation given by equation (22) with $\hat{\gamma} \gtrsim 1$. Such particles with $\hat{\gamma}$ near unity provide an ambient medium surrounding the central source. Highenergy particles originating near the poles of the central collapsed configuration and moving outward along the rotation axis faster than the critical speed $1 / \sqrt{2} \approx 0.7$ relative to the ambient medium experience significant tidal deceleration ${ }^{25}$, while such ultrarelativistic particles moving outward normal to the rotation axis experience significant tidal acceleration relative to the ambient medium. In a series of recent papers, these phenomena have been investigated in some detail. ${ }^{24-27}$ It turns out that tidal deceleration occurs for outflow velocities within a cone of half angle $\theta \approx \tan ^{-1} \sqrt{2} \approx 55^{\circ}$ around the rotation axis. ${ }^{26,27}$ On the other hand, tidal acceleration occurs for outflow velocities outside the critical velocity cone. ${ }^{26,27}$

A complete analysis must of course include plasma and radiation effects as well. Nevertheless, preliminary results appear to be consistent with observations of microquasars and Chandra studies of certain neutron star $X$-ray sources in our Galaxy. ${ }^{25-27}$ 


\section{Discussion}

Using the main approaches to GEM , we have demonstrated the existence of a critical speed that could have fundamental consequences for the gravitational physics of ultrarelativistic flows. The physical origin of the critical speed can be traced back to a "conflict" between the intuitively expected behavior of particles based on Newtonian gravitation and the behavior of light in a gravitational field; indeed, both of these aspects are properly integrated within general relativity.

Imagine a light ray propagating from event $P_{1}:\left(t_{1}, \mathbf{x}_{1}\right)$ to event $P_{2}:\left(t_{2}, \mathbf{x}_{2}\right)$ in a global inertial system of reference; hence, $t_{2}-t_{1}=\left|\mathbf{x}_{2}-\mathbf{x}_{1}\right|$. If the spacetime is now slightly perturbed by the presence of a GEM field as in Section 2, then the arrival of the signal at $P_{2}$ is accompanied mainly by a Shapiro gravitoelectric delay given by

$$
\Delta_{G E}=2 \int_{P_{1}}^{P_{2}} \Phi d \ell
$$

where $d \ell$ is the element of length along the path of the unperturbed ray. ${ }^{28}$ This is intimately related to the fact that the propagation of electromagnetic waves in the gravitational medium takes place with an effective index of refraction given by $^{29} n \approx 1+2 \Phi$ for $\Phi<<1$. On the other hand, the kinetic energy of a particle increases as it gets closer to a gravitational source according to the Newtonian theory of gravitation. The conflict is resolved in general relativity through the existence of a critical speed such that "ultrarelativistic" particle motion with speed above this critical speed has significant features that violate our nonrelativistic intuition based on Newtonian gravitation. In the linear post-Newtonian approach, the critical coordinate speed is $1 / \sqrt{3}$; however, for relative motion in Fermi coordinates, the invariant critical speed is $1 / \sqrt{2}$. The latter case has been the subject of recent studies due to its direct observational significance.

The most significant consequence of the existence of the critical speed is a possible gravitational mechanism for the acceleration/deceleration of ultrarelativistic particles. This mechanism can be elucidated by comparing and contrasting the gravitational and electromagnetic interactions. The motion of a charged particle in an external electromagnetic field in Minkowski spacetime is given by

$$
\frac{d}{d t}\left(\frac{\mathbf{v}}{\sqrt{1-v^{2}}}\right)=\frac{q}{m}(\mathcal{E}+\mathbf{v} \times \mathcal{B})
$$

in accordance with the Lorentz force law. This equation may be written as

$$
\frac{d \mathbf{v}}{d t}=\frac{q}{m} \sqrt{1-v^{2}}[\mathcal{E}-\mathbf{v}(\mathbf{v} \cdot \mathcal{E})+\mathbf{v} \times \mathcal{B}]
$$

which indicates that the closer $v$ gets to unity, the more difficult it will be to change the velocity of the particle. For a finite external electromagnetic field, the motion of a charged particle with $m \neq 0$ is effectively uniform for $v$ approaching unity. Thus the critical speed that appears in the electromagentic case is the fundamental speed, namely, unity. 
The gravitational analog of equation (40) in the quasi-inertial Fermi coordinate system is

$$
\frac{d}{d T}\left(\Gamma V^{i}\right)=-\Gamma\left({ }^{F} \Gamma_{00}^{i}+2{ }^{F} \Gamma_{0 j}^{i} V^{j}+{ }^{F} \Gamma_{j k}^{i} V^{j} V^{k}\right) .
$$

while the analog of equation (41) is given by equation (32). The fact that the right-hand side of equation (42) is proportional to the modified Lorentz factor $\Gamma$ is responsible for the bending of ultrarelativistic particle orbits by a gravitational field-indeed, this deflection approaches that of light in the appropriate limit. Similarly, equation (32) implies that it is in general possible to change the velocity of ultrarelativistic particles considerably in a gravitational field, since the analog of $\sqrt{1-v^{2}}$ in equation (41) involving the fundamental speed is missing in equation (32); indeed, there is a critical speed of $1 / \sqrt{2}$, but it appears in a different way. An even more striking illustration of this circumstance can be given for onedimensional motion.

Consider a situation where $\mathcal{E}$ and $\mathcal{B}$ are parallel and the charged particle moves along the field lines. Then equation (41) reduces to

$$
\frac{d \mathbf{v}}{d t}=\frac{q}{m}\left(1-v^{2}\right)^{3 / 2} \mathcal{E},
$$

which may be compared and contrasted with equations (18) and (38). While the ultimate ("critical") speed in equation (43) severely restricts the magnitude of acceleration of a particle with $v \rightarrow 1$, this is not the case for gravitational motion in equations (18) and (38), which exhibit instead critical speeds of $1 / \sqrt{3}$ and $1 / \sqrt{2}$, respectively.

The appearance of the critical factor $\left(1-2 \dot{Z}^{2}\right)$ in equation (38) is due to the fact that in the quasi-inertial Fermi coordinates, one finds that to lowest order

$$
{ }^{F} \Gamma_{00}^{i}={ }^{F} \Gamma_{0 i}^{0}=-\frac{1}{2}\left({ }^{F} g_{00}\right)_{, i} .
$$

Indeed, the first term in the critical factor originates with ${ }^{F} \Gamma_{00}^{i}$, while the second term originates with ${ }^{F} \Gamma_{0 i}^{0}$. The factor 2 in the latter term is due to the fact that ${ }^{F} \Gamma_{0 i}^{0}$ occurs twice, since ${ }^{F} \Gamma_{0 i}^{0}={ }^{F} \Gamma_{i 0}^{0}$; hence, the tensorial character of general relativity is responsible for the appearance of the factor of 2 in $\left(1-2 \dot{Z}^{2}\right)$. Thus the circumstance that the critical speed is unity in electrodynamics while it is $1 / \sqrt{2}$ in gravitation is related to the spin- 1 nature of the electromagnetic field and the spin-2 nature of the linearized general relativity in the Fermi system, respectively.

Recent investigations of the novel gravitational mechanism for the acceleration/deceleration of ultrarelativistic particles indicate that this mechanism may play a fundamental role in high-energy astrophysics. ${ }^{24-27}$ Furthermore, the tidal motion of charged particles has also been recently examined in an interesting astrophysical context in Ref. 30, where the important role of the critical speed has been independently confirmed. 


\section{Appendix A. Gravitational Dynamics of Relativistic Flows}

Imagine a perfect fluid with energy density $\rho$ and pressure $p$ in the gravitational field considered in Section 4. The energy-momentum tensor of the fluid is given by

$$
T^{\mu \nu}=(\rho+p) u^{\mu} u^{\nu}+p g^{\mu \nu}
$$

where $u^{\mu}$ is the unit four-velocity vector of the fluid. The purpose of this Appendix is to express the dynamical laws $T_{; \nu}^{\mu \nu}=0$ in the Fermi coordinate system established about the worldline of the geodesic observer $\mathcal{O}$.

Let us first note that in a global inertial frame of reference, the dynamical laws may be expressed as

$$
\begin{aligned}
\frac{\partial \tilde{\rho}}{\partial t}+\nabla \cdot(\tilde{\rho} \mathbf{v}) & =\frac{\partial p}{\partial t}, \\
\tilde{\rho} \frac{d \mathbf{v}}{d t} & =-\nabla p-\frac{\partial p}{\partial t} \mathbf{v},
\end{aligned}
$$

where $\tilde{\rho}=\gamma^{2}(\rho+p)$ and $\gamma=\left(1-v^{2}\right)^{-1 / 2}$ is the Lorentz factor of the fluid. Equation (A.2) follows directly from the $\mu=0$ component of $T_{; \nu}^{\mu \nu}=0$, while the $\mu=i$ component, when combined with equation (A.2) implies equation (A.3). Equations (A.2) and (A.3) are the relativistic continuity and Euler equations, respectively, and assume their familiar form when the pressure has no explicit dependence upon time.

In the quasi-inertial Fermi coordinates, we expect that the dynamical laws would have the same form as equations (A.2) and (A.3) but modified by the presence of curvature terms. In fact, let the four-velocity be $\Gamma(1, \mathbf{V})$ as before, where $\Gamma>0$ is given by equation (30). Then with $\tilde{\rho}=\Gamma^{2}(\rho+p)$, the modified equations of motion become

$$
\begin{aligned}
\frac{\partial \tilde{\rho}}{\partial T}+\nabla \cdot(\tilde{\rho} \mathbf{V}) & =-p_{, \alpha}{ }^{F} g^{0 \alpha}-\tilde{\rho} K \\
\tilde{\rho}\left[\frac{d V^{i}}{d T}+\left({ }^{F} \Gamma_{\alpha \beta}^{i}-{ }^{F} \Gamma_{\alpha \beta}^{0} V^{i}\right) \frac{d X^{\alpha}}{d T} \frac{d X^{\beta}}{d T}\right] & =-p_{, \alpha}{ }^{F} g^{i \alpha}+\left(p_{, \alpha}{ }^{F} g^{0 \alpha}\right) V^{i},
\end{aligned}
$$

where

$$
K=\left({ }^{F} \Gamma_{\alpha \beta}^{0} \frac{d X^{\alpha}}{d T}+{ }^{F} \Gamma_{\alpha \beta}^{\alpha}\right) \frac{d X^{\beta}}{d T} .
$$

To lowest order, $K$ is given by

$$
K=\frac{1}{3}\left(8^{F} R_{0 i 0 j}-{ }^{F} R_{i j}\right) V^{i} X^{j}+\frac{2}{3}{ }^{F} R_{0 i j k} V^{i} V^{j} X^{k} .
$$

It follows from these results that equations (A.2) and (A.3) are recovered along the reference trajectory at $(T, \mathbf{0})$, as required. Moreover, in the absence of pressure equation (A.5) is equivalent to equation (32) with attendant consequences regarding the existence of the critical speed $V_{c}=1 / \sqrt{2}$. A complete analysis of equations (A.4) and (A.5) is beyond the scope of this work. 


\section{References}

1. J.C. Maxwell, Phil. Trans. Roy. Soc. London CLV (1865), §82.

2. G. Holzmüller, Z. Math. Phys. 15, 69 (1870).

3. F. Tisserand, Compt. Rend. 75, 760 (1872); 110, 313 (1890).

4. A. Einstein, The Meaning of Relativity (Princeton University Press, Princeton, 1950).

5. W. de Sitter, Mon. Not. Roy. Astron. Soc. 76, 699 (1916).

6. W.W. Salisbury and D.H. Menzel, Nature 252, 664 (1974).

7. H. Thirring, Phys. Z. 19, 33 (1918); 22, 29 (1921).

8. J. Lense and H. Thirring, Phys. Z. 19, 156 (1918).

9. B. Mashhoon, F.W. Hehl and D.S. Theiss, Gen. Rel. Grav. 16, 711 (1984).

10. Reference Frames and Gravitomagnetism, edited by J.-F. Pascual-Sánchez, L. Floría, A. San Miguel and F. Vicente (World Scientific, Singapore, 2001).

11. Nonlinear Gravitodynamics, edited by R.J. Ruffini and C. Sigismondi (World Scientific, Singapore, 2003).

12. The Measurement of Gravitomagnetism: A Challenging Enterprise, edited by L. Iorio (NOVA Science, Hauppauge, New York, 2006).

13. 〈http://www.gravityprobeb.com〉

14. Gyros, Clocks, Interferometers...: Testing Relativistic Gravity in Space, edited by C. Lämmerzahl, C.W.F. Everitt and F.W. Hehl, Lect. Notes Phys. 562 (Springer, Berlin, 2001).

15. C.W.F. Everitt et al., in Near Zero: Festschrift for William M. Fairbank, edited by C.W.F. Everitt (Freeman, San Francisco, 1986).

16. B. Mashhoon, Phys. Lett. A 173, 347 (1993).

17. B. Mashhoon, L. Iorio and H. Lichtenegger, Phys. Lett. A 292, 49 (2001).

18. M. Carmeli, Nuovo Cimento Lett. 3, 379 (1972); Classical Fields: General Relativity and Gauge Theory (Wiley, New York, 1982), pp. 230-234.

19. J. Jaffe and I.I. Shapiro, Phys. Rev. D 6, 405 (1972).

20. F.S. Felber, gr-qc/0505098; gr-qc/0505099.

21. S.I. Blinnikov, L.B. Okun and M.I. Vysotsky, Phys. Usp. 46, 1099 (2003) [Usp. Fiz. Nauk. 46, 1131 (2003)].

22. J.L. Synge, Relativity: The General Theory (North-Holland, Amsterdam, 1960).

23. B. Mashhoon, J.C. McClune and H. Quevedo, Phys. Lett. A 231, 47 (1997); Class. Quantum Grav. 16, 1137 (1999).

24. C. Chicone and B. Mashhoon, Class. Quantum Grav. 19, 4231 (2002); 21, L139 (2004); 22, 195 (2005).

25. C. Chicone, B. Mashhoon and B. Punsly, Int. J. Mod. Phys. D 13, 945 (2004); Phys. Lett. A 343, 1 (2005).

26. C. Chicone and B. Mashhoon, Ann. Phys. (Leipzig) 14, 290 (2005).

27. C. Chicone and B. Mashhoon, Astron. \& Astrophys. 437, L39 (2005).

28. I. Ciufolini, S. Kopeikin, B. Mashhoon and F. Ricci, Phys. Lett. A 308, 101 (2003).

29. B. Mashhoon, Phys. Rev. D 7, 2807 (1973); 10, 1059 (1974).

30. Y. Kojima and K. Takami, gr-qc/0509084. 